\title{
Improved Nyquist Pulse Shaping Filters for Generalized Frequency Division Multiplexing
}

\author{
Atul kumar and Maurizio Magarini \\ Dipartimento di Elettronica, Informazione e Bioingegneria \\ Politecnico di Milano \\ Milano, Italy \\ \{atul.kumar,maurizio.magarini $\} @$ polimi.it
}

\begin{abstract}
Generalized Frequency-Division Multiplexing (GFDM) is one of the multicarrier modulation schemes currently under study for next generation 5G cellular networks. One of the main characteristics of GFDM is the low out of band emission that is achieved by means of a flexible time-domain pulse shaping of individual subcarriers. In the paper, we propose to use improved Nyquist pulse shaping filters which have been originally introduced in the context of single-carrier modulation schemes for reducing the sensitivity to symbol timing error due to their higher eye opening and smaller maximum distortion. Here we consider their use in GFDM and evaluate their symbol error rate (SER) performance in case of 16-QAM transmission over an additive white Gaussian noise channel. Moreover, we also considered the concept of the wavelet for better time-frequency localization of the pulse shaping filters by using the Meyer auxiliary function. Numerical results are reported to demonstrate the superior SER performance achieved by the proposed improved Nyquist pulse shaping filters in comparison to that achieved with conventional Nyquist pulse shaping filters.
\end{abstract}

Keywords - Generalized frequency-division multiplexing (GFDM); Nyquist filtering; symbol error rate (SER).

\section{INTRODUCTION}

Nowadays, increasing demand for smart devices with low round-trip latency and ultra-high reliability appears as a decisive factor for the implementation of future communication systems [1]. Upcoming $5^{\text {th }}$ generation $(5 \mathrm{G})$ cellular networks must be able to cope with a high number of connected devices as well as a significant growth in data volume [2]. Main scenarios for 5G cellular networks are machine type communication (MTC) [3], Tactile Internet [4] and wireless regional area network (WRAN) [5]. In particular, the introduction of MTC, characterized by devices with sporadic random access, can be accomplished by means of cognitive radio (CR) techniques [6], where the flexibility in shaping and structuring the transmit signal is a key aspect.

A CR system must be able to flexibly aggregate spectrum white spaces where, at the same time, the out-of-band (OOB) leakage is minimized in order not to affect neighboring systems. As is well known, current cellular transmission schemes like Long-Term Evolution (LTE) and LTE-Advanced (LTE-A) employ orthogonal frequency division multiplexing (OFDM) in the PHY layer [7]. Although OFDM is robust against the frequency-selective fading channel and offers an efficient implementation using the Fourier transform, it is not well suited for future requirements [8]. Main weaknesses of OFDM are its sensitivity to carrier frequency offset and phase noise [9], [10], [11] and its large OOB leakage, which makes it unattractive for CR applications. While effects of carrier frequency offset and phase noise can be mitigated by fine synchronization and phase tracking capabilities [12], [13], a reduction of OOB emission can be achieved through a time-domain pulse shaping of individual subcarriers. In fact, high OOB radiation of OFDM is due to the rectangular pulse shaping filter applied on each subcarrier in time domain, which leads to large tails in the frequency domain. Another problem of OFDM is the requirement of a cyclic prefix (CP) in every symbol which reduces the spectral efficiency. In order to address all these issues, new multi-carrier modulation (MCM) schemes are under investigation for the 5G physical layer. Among the proposed 5G MCM schemes, filter bank multicarrier (FBMC) [14] and generalized frequency division multiplexing (GFDM) [15] are the most promising candidates for $5 \mathrm{G}$ application.

In FBMC modulation the use of a prototype pulse shaping filter on each subcarrier is proposed to minimize OOB radiation. Several works in the literature focus on the design of pulse shaping filters for FBMC with the aim of minimizing the OOB radiation [16], [17]. The main property of FBMC is that of maintaining orthogonality among sub-carriers and allowing for reduction of $\mathrm{OOB}$ emissions by a proper choice of the pulse shaping filters. However, it does not have some other properties that are needed in 5G such as robustness against the frequency selective fading channel. In contrast, GFDM that is derived from the filter bank approach, is based on a block filtered MCM scheme allows to achieve higher robustness to frequency selective fading channel. The data transmission on each block is distributed in time and frequency and each sub-carrier is pulseshaped. Reduction of OOB emission is obtained in GFDM by using different time-domain pulse shaping filters other than the conventional rectangular one used in CP-OFDM [18]. Moreover, the tail biting technique used in GFDM makes the CP length to be independent of transmit and receive filters length [16]. This allows to reduce the overhead caused by the $\mathrm{CP}$, thus increasing the spectral efficiency. Another advantage of GFDM is related to its flexible frame structure: by changing the number of time slots or sub-carriers in a GFDM frame, it can cover both conventional CP-OFDM and single-carrier frequency-domain multiple access. Furthermore, GFDM allows to achieve an additional frequency diversity gain at the cost of an inherent self- 


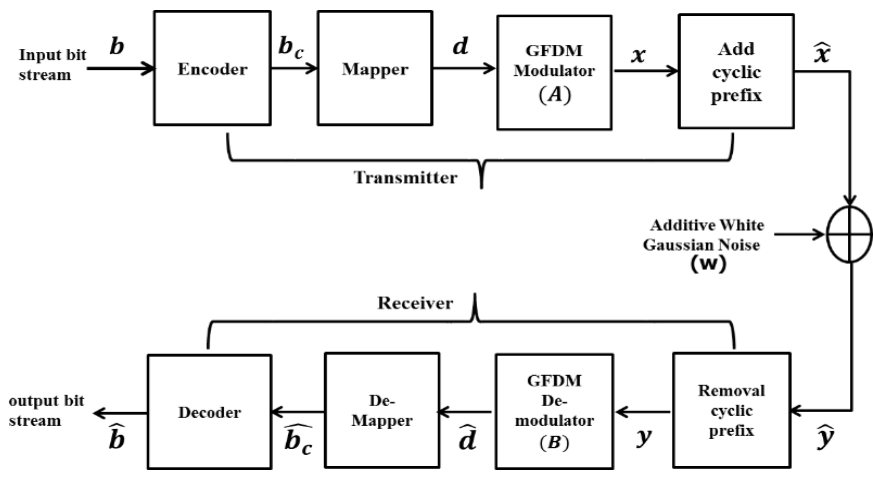

Figure 1: Block diagram of baseband GFDM transceiver.

interference that originates from the non-orthogonality and, therefore, prohibits the decoupling of individual subcarriers. These features make GFDM the strongest candidate MCM scheme for $5 \mathrm{G}$.

Several standard receiver options can be used for demodulation of the GFDM signal:

- Matched filter (MF): MF receiver maximizes the signal-tonoise ratio (SNR) per subcarrier, but with the effect of introducing self-interference when a non-orthogonal transmit pulse is applied. Methods for the cancellation of self-interference with a MF receiver have been proposed in [19], where it is shown that almost equal performance as orthogonal MCM can be achieved. The same MF receiver is considered in [18] to study the impact of different pulse shaping filters on OOB and error rate performance.

- Linear minimum mean-squared error (MMSE): MMSE receiver makes a trade-off between self-interference and noise enhancement.

- Zero forcing (ZF): ZF receiver completely removes selfinterference at the receiving side at the cost of introducing a possible noise enhancement, which depends on the impulse response of the pulse shaping filter. Noise enhancement has an impact on symbol error-rate (SER) performance. So, by a proper design of the pulse shaping it is possible to overcome the effect of noise enhancement and to improve the SER performance.

The main contribution of this paper consists in the evaluation of the SER performance for GFDM using different types of improved Nyquist pulse shaping filters. Transmission over an additive white Gaussian noise (AWGN) channel is considered in the case of ZF receiver. Improved Nyquist pulse shaping filters have been originally proposed in [20] to achieve higher tolerance to symbol timing error in single-carrier modulation schemes. As suggested in [21], when Nyquist pulse shaping filters are considered in GFDM a performance improvement can be achieved by exchanging the role of the frequency independent variable with that of the time independent variable in the formal expression defining the raised cosine pulse. The main advantage is that there is no overlapping in the frequency domain and, therefore, less interference is observed.
The paper is organized as follows. Section II gives a short description of the GFDM system model together with the notation that will be used throughout this paper. The different types of improved Nyquist pulse shaping filters are introduced in Sec. III, while the SER analysis of GFDM is provided in Sec. IV. Simulation results for different pulse shaping filters are given in Sec. V and, finally, conclusions are drawn in Sec. VI.

\section{SYSTEM MODEL}

The block diagram of the considered GFDM transmission scheme is shown in Figure 1. In this scheme the transmitter part includes several blocks. By using a matrix notation, the vector of input bits $\mathbf{b}$ is applied at the input of the encoder. The encoder splits the high bit-rate stream into a number of lower bit-rate streams and forms the encoded vector $\mathbf{b}_{c}$. The vector $\mathbf{b}_{c}$ is applied at the input of the mapper which gives at its output an $N \times 1$ data vector $\mathbf{d}$ whose elements take values from a complex constellation, e.g., QAM. The data vector $\mathbf{d}$ is then applied at the input of the GFDM modulator that contains $N$ elements. Which can be decomposes into $K$ groups of $M$ symbols according to

with

$$
\mathbf{d}=\left[\left(\boldsymbol{d}_{0}\right)^{T},\left(\mathbf{d}_{1}\right)^{T}, \ldots \ldots,\left(\mathbf{d}_{K-1}\right)^{T}\right]^{T}
$$

$$
\mathbf{d}_{k}=\left[d_{k, 0}, d_{k, 1}, \ldots . ., d_{k, M-1}\right]^{T},
$$

where $d_{k, m}$ corresponds to the QAM symbol transmitted on the $k$-th sub-carrier and in the $m$-th sub-symbol of the block and $(\cdot)^{T}$ denotes transposition. The time-duration of each data block $\mathbf{d}_{k}$ is $M T_{s}$ and the sub-carrier spacing is equal to $1 / M T_{s}$.

In the GFDM modulator, each $d_{k, m}$ is transmitted with the corresponding pulse shape

$$
g_{k, m}[n]=g[(n-m k) \bmod N] e^{\frac{-j 2 \pi k n}{K}},
$$

where $g[n]$ is a prototype discrete-time impulse response and $n$ is the sampling index. Note that, each $g_{k, m}[n]$ corresponds to a time and frequency shifted version of $g[n]$, where the complex exponential performs the shifting operation in the frequency domain. The $n$-th entry of the $M K \times 1$ transmitted vector $\mathbf{x}$ is given by

$$
x[n]=\sum_{k=0}^{K-1} \sum_{m=0}^{M-1} d_{k, m} g_{k, m}[n], \quad n=0,1, \ldots \ldots . . K M-1 .
$$

By collecting the pulse shaping filter samples in the $K M \times 1$ vector $\boldsymbol{g}_{k, m}=\left[g_{k, m}[0], g_{k, m}[1], \ldots, g_{k, m}[M K-1]\right]^{T}$, allows to represent eq. (2) in matrix form as

$$
\mathbf{x}=\text { Ad, }
$$

where $\mathbf{A}$, a $K M \times K M$ matrix known as GFDM modulation matrix is given by [22]

$$
\mathbf{A}=\left[\begin{array}{lllll}
\boldsymbol{g}_{0,0} \ldots & \boldsymbol{g}_{K-1,0} & \boldsymbol{g}_{0,1} \ldots \ldots & \boldsymbol{g}_{K-1,1} & \boldsymbol{g}_{0, M-1} \ldots \\
\boldsymbol{g}_{K-1, M-1}
\end{array}\right]
$$

Before transmission a CP of length $N_{C P}$ is added to form the vector $\widehat{\boldsymbol{x}}=\left[\mathbf{x}\left(N-N_{C P}: N-1\right)^{T}, \mathbf{x}^{T}\right]^{T}$. 
Considering transmission over an AWGN channel, the received signal vector is modelled as

$$
\widehat{\boldsymbol{y}}=\widehat{\boldsymbol{x}}+\mathbf{w},
$$

where each entry of $\mathbf{w}$ is an i.i.d. zero mean complex Gaussian random variable with variance $\sigma_{w}^{2}$. The received vector after removal of the CP is defined as $\mathbf{y}=\widehat{\boldsymbol{y}}\left(N_{C P}: N_{C P}+N-1\right)$. After weighting with the ZF matrix $\mathbf{B}_{Z F}=\mathbf{A}^{-1}$, the received signal vector is given by

$$
\mathbf{z}=\mathbf{B}_{Z F} \mathbf{A d}+\mathbf{B}_{Z F} \mathbf{w}=\mathbf{d}+\widetilde{\mathbf{w}} .
$$

From the eq. (6) we observe that the ZF receiver completely removes self-interference at the cost of noise enhancement. The noise enhancement factor (NEF) $\xi$ determines the SNR reduction when using the $\mathrm{ZF}$ receiver. It is defined as

$$
\xi=\sum_{n=0}^{M K-1}\left|\left[B_{Z F}\right] k, n\right|^{2},
$$

which is the same for every $k$.

\section{PULSE SHAPING FILTER}

The choice of the pulse shaping filters $g[\cdot]$ strongly influences the spectral properties of the GFDM signal. As is well known, Nyquist impulse responses have periodic zero values in time-domain at multiples of the symbol period. When we move in the frequency domain this condition turns into a vestigial symmetry [23]. This means that their spectrum exhibits odd symmetry around the cut-off frequency, which is half the symbol rate. Hence, Nyquist filters can be defined by imposing an odd symmetry to the frequency coefficients around the cutoff frequency. In digital communication systems, the global Nyquist filter is generally split into two parts, a square-root Nyquist filter in the transmitter and a square-root Nyquist filter in the receiver. Then, the symmetry condition is satisfied by the squares of the frequency coefficients. In order to design a proper Nyquist filter to be used in GFDM, the principles of odd symmetry need to be applied from a time-domain perspective by interchanging the role of the frequency independent variable with that of the time independent variable in the analytical function defining the Nyquist function. The advantage deriving from this formal exchange between the two independent variables is the elimination of the time-domain overlapping with consequent reduction of interference.

The concept of wavelet is here included to achieve a better time-frequency localization and also to add flexibility in the design of the pulse shaping filter. As is well known, square-root Nyquist pulse has a major impact on the system design and, therefore, an alternative solution to define it in the time-domain is required. This alternative solution must assure that the derivative of the designed pulse is smooth in order to confine the frequency span as much as possible. Many authors have investigated solutions based on the concept of wavelets [24, 25]. A wavelet is a wave-like oscillation with an amplitude that begins at zero, increases, and then decreases back to zero. To assure this smooth transition, an auxiliary function was introduced by Meyer in [26] to be used as an inner argument of different pulse shaping filters. The Meyer auxiliary function is defined as

$$
v(x)=x^{4}\left(35-84 x+70 x^{2}-20 x^{3}\right),
$$

Therefore, in our results we will consider the impact of improved Nyquist pulse shaping filters with and without Meyer on the SER performance of the GFDM in case of transmission over the AWGN channel.

\section{A. Root raised cosine (RRC)}

This category of pulse shaping filters is defined by the wellknown raised cosine (RC) and root raised cosine (RRC) functions in time-domain for a given roll-off factor $\alpha$. Here, we consider the time-domain expression that results by interchanging the independent frequency variable with the time variable

$$
g_{R C}(t)=\left\{\begin{array}{cc}
1, & |t| \leq \frac{(1-\alpha) T}{2} \\
\frac{1}{2}\left[1+\cos \left(\pi P_{R C}(t)\right)\right], & \frac{(1-\alpha) T}{2}<|t| \leq \frac{(1+\alpha) T}{2} \\
0, & \text { otherwise, }
\end{array}\right.
$$

where $P_{R C}(t)$ is the inner argument of the cosine written as

$$
P_{R C}(t)=\left(\frac{|t|-\left(\frac{(1-\alpha) T}{2}\right)}{\alpha T}\right) .
$$

By introducing the Meyer auxiliary function $v(x)$, the inner argument of the cosine must be modified as

$$
P_{R C}(t)=v\left(\frac{|t|-\left(\frac{(1-\alpha) T}{2}\right)}{\alpha T}\right) .
$$

The RRC pulse shaping filter is defined as

$$
g_{R R C}(t)=\sqrt{g_{R C}(t)} .
$$

\section{B. Flipped-hyperbolic secant (Fsech)}

The improved Nyquist pulse shaping filter proposed in [20], by following [20], first we consider the flipped-hyperbolic secant (Fsech) pulse shaping filters. The time-domain expression is obtained by interchanging independent frequency variable with the time variable is given by

$$
g(t)=\left\{\begin{array}{rr}
1, & |t| \leq \frac{(1-\alpha) T}{2} \\
1-\operatorname{sech}\left(\rho P_{1_{\text {Fsech }}}(t)\right), & \frac{(1-\alpha) T}{2}<|t| \leq \frac{T}{2} \\
\operatorname{sech}\left(\rho P_{2_{\text {Fsech }}}(t)\right), & \frac{T}{2}<|t| \leq \frac{(1+\alpha) T}{2} \\
0, & \frac{(1+\alpha) T}{2}<|t|
\end{array}\right.
$$


where sech is the hyperbolic secant function, $\rho=\ln (\sqrt{3}+2) /$ $\alpha \times \frac{T}{2}, P_{1_{\text {Fsech }}}(t)$ and $P_{2_{\text {Fsech }}}(t)$ is the inner arguments of the hyperbolic secant function written as

$$
P_{1_{F s e c h}}(t)=\left(\left(\frac{(1+\alpha) T}{2}\right)-|t|\right)
$$

and

$$
P_{2_{\text {Fsech }}}(t)=\left(|t|-\left(\frac{(1-\alpha) T}{2}\right)\right) \text {. }
$$

When we introduce the Meyer auxiliary function $v(x)$, inner arguments of the hyperbolic secant function $P_{1_{\text {Fsech }}}(t)$ and $P_{2_{\text {Fsech }}}(t)$ must be modified as

$$
P_{1_{\text {Fsech }}}(t)=v\left(\left(\frac{(1+\alpha) T}{2}\right)-|t|\right),
$$

and

$$
P_{2_{F s e c h}}(t)=v\left(|t|-\left(\frac{(1-\alpha) T}{2}\right)\right) .
$$

\section{Flipped-inverse hyperbolic secant (Farcsech)}

Another considered improved Nyquist pulse shaping filter is that proposed in [20] that is known as flipped-inverse hyperbolic secant (Farcsech). The time-domain expression of Farcsech pulse shaping filters is obtained by interchanging independent frequency variable with the time variable is given by

$$
g(t)=\left\{\begin{array}{lr}
1, & |t| \leq \frac{(1-\alpha) T}{2} \\
\operatorname{arcsech}\left(\frac{1}{\rho} P_{1_{\text {Farcsech }}}(t)\right), & \frac{(1-\alpha) T}{2}<|t| \leq \frac{T}{2} \\
1-\operatorname{arcsech}\left(\frac{1}{\rho} P_{2_{\text {Fsech }}}(t)\right), \frac{T}{2}< & |t| \leq \frac{(1+\alpha) T}{2} \\
0, & \frac{(1+\alpha) T}{2}<|t|
\end{array}\right.
$$

where arcsech is the inverse hyperbolic secant function, $P_{1_{\text {Farcsech }}}(t)$ and $P_{2 \text { Fsech }}(t)$ is the inner arguments of the inverse hyperbolic secant function written as

$$
P_{1_{\text {Farcsech }}}(t)=\left(|t|-\left(\frac{(1-\alpha) T}{2}\right)\right),
$$

and

$$
P_{2_{F s e c h}}(t)=\left(\left(\frac{(1+\alpha) T}{2}\right)-|t|\right) .
$$

By introducing the concept of the Meyer auxiliary function $v(x)$, the inner arguments Farcsech pulse shaping filters $P_{1_{\text {Farcsech }}}(t)$ and $P_{2 \text { Fsech }}(t)$ are modified as and

$$
P_{1_{\text {Farcsech }}}(t)=v\left(\left(\frac{(1+\alpha) T}{2}\right)-|t|\right)
$$

$$
P_{2_{\text {Farsech }}}(t)=v\left(|t|-\left(\frac{(1-\alpha) T}{2}\right)\right)
$$

\section{SER ANALYSIS}

In this section, we analyze the SER performance of the GFDM system in the case of the ZF receiver. This means that self-generated interference is removed on the receiver side but noise enhancement can be introduced, depending on the pulse shaping filters. The SER performance is evaluated considering the AWGN channel for16-QAM modulation technique by considering different improved Nyquist pulse shaping filters given in [20]. Moreover, we have also considered the concept of wavelet to achieve better time-frequency localization of the pulse shaping filters, by using the Meyer auxiliary function. A comparison of the GFDM SER performance is done for different improved Nyquist pulse shaping filters with and without using Meyer auxiliary functions.

The NEF adjusts the equivalent SNR for GFDM at the receiver side [22]. Therefore, the SER expression of 16-QAM modulation technique for GFDM system transmission over the AWGN is written as

$$
P_{A W G N}=2\left(\frac{\kappa-1}{\kappa}\right) \operatorname{erfc}(\sqrt{\gamma})-\left(\frac{\kappa-1}{\kappa}\right) \operatorname{erfc}^{2}(\sqrt{\gamma})
$$

where, $\gamma=\frac{3 R_{T}}{2\left(2^{\mu}-1\right)} \frac{E_{S}}{\xi N_{o}}, R_{T}=\frac{K M}{K M+N_{C p}+C_{C S}}, \mu$ is the number of bits per QAM symbol, $\kappa=\sqrt{2^{\mu}}$, and $N_{c p} \& N_{c s}$ are the length of CP and guard symbol, respectively, $E_{s}$ is the average energy per symbol, and $N_{o}$ is the noise power density of complex AWGN. The SER performance of GFDM is severely degraded due to the noise enhancement and, therefore, the prototype pulse shape must be properly chosen in order to avoid prohibitive performance loss due to the NEF.

\section{Simulation Results}

Figure 2 shows the time-domain plots of different Nyquist pulse shaping filters such as RRC, Fsech and Farcsech for different values of roll-off $(\alpha)=0.1,0.5$ and 0.5 and the corresponding time-domain filter expressions are obtained by interchanging the frequency variable with the time variable. After interchanging the independent variable, the corresponding time-domain expression of RRC, Fsech and Farcsech pulse shaping filters are given in the eqns. (9), (13) and (18) respectively. From the Fig. 2, it is clearly visible that by increasing the value of $\alpha$ we have larger spread but in case of Farcsech pulse shape filter we have almost same band at different values of $\alpha$.

Figure 3 shows the comparison of SER performance verses $E_{s} / N_{o}$ of 16-QAM with different pulse shaping filters given by eqns. (9), (13) and (18) with and without Meyer for the GFDM system transmission over the AWGN channel. A number of 64 


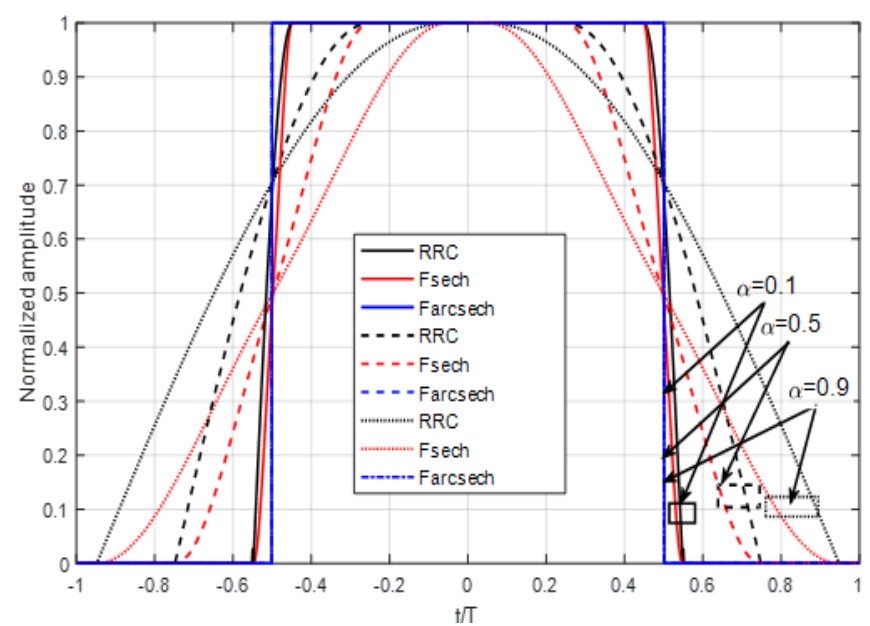

Figure 2: Time-domain function of different pulse shaping filters.

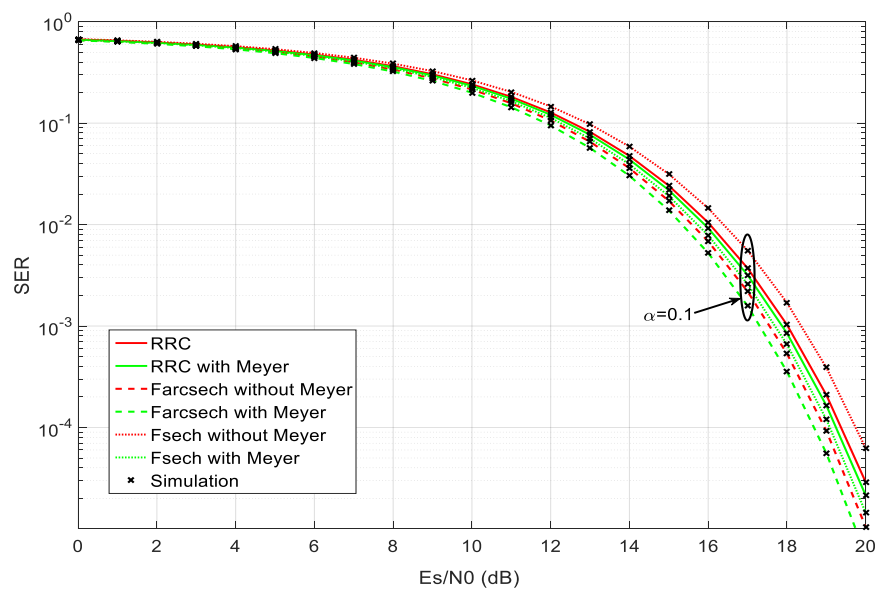

Figure 3: SER performance of different pulse shaping filter at $\alpha=0.1$.

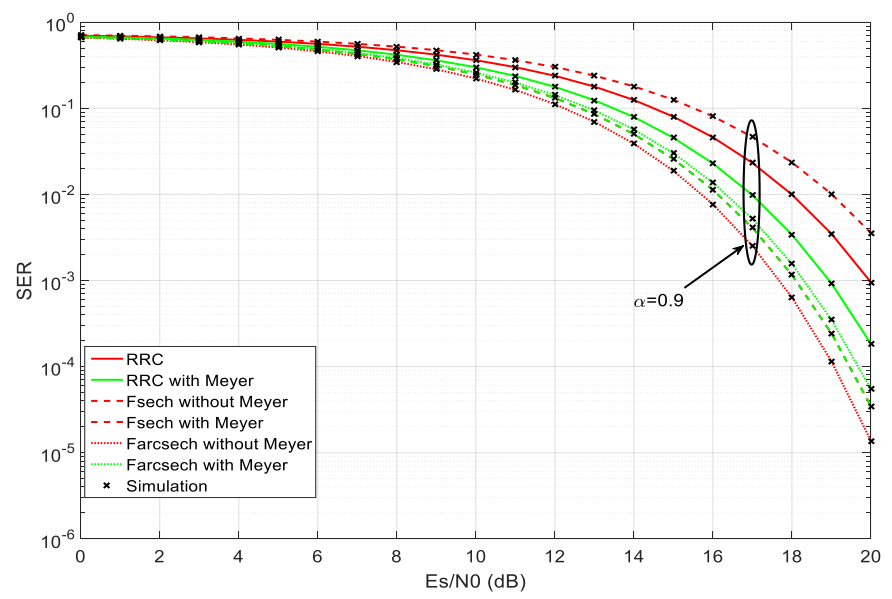

Figure 4: SER SER performance of different pulse shaping filter at $\alpha=0.9$.

sub-carriers is considered where on each sub-carrier contain the 7 sub-symbols with $\alpha=0.1$. It is clearly evident from the graphs that at $\alpha=0.1$, Farcsech pulse shaping filter is the optimal one in comparison with the RRC given in [22] and with Fsech pulse shaping filter.

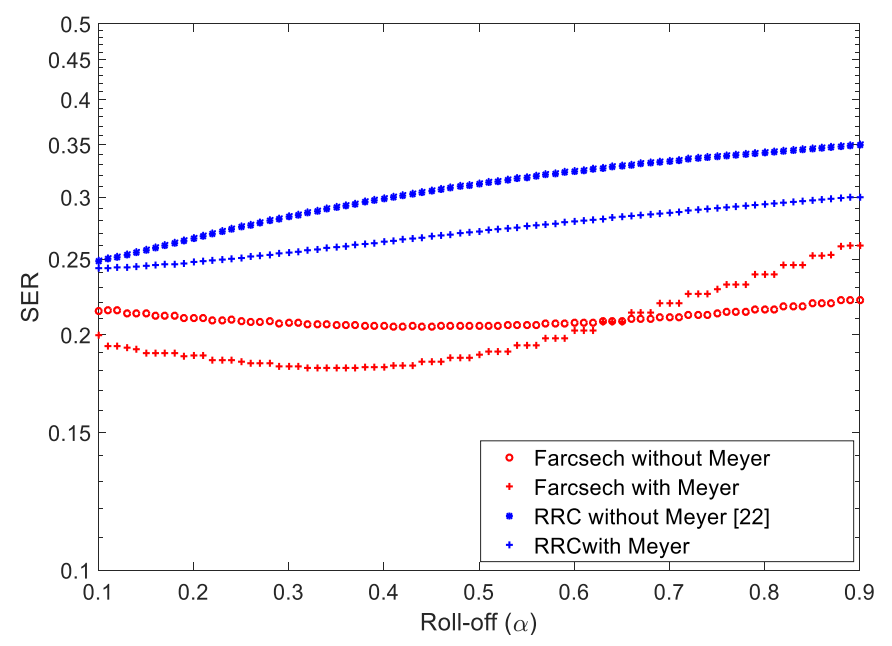

Figure 5: SER comparison of RRC and Farcsech with and without Meyer at 10 dB SNR.

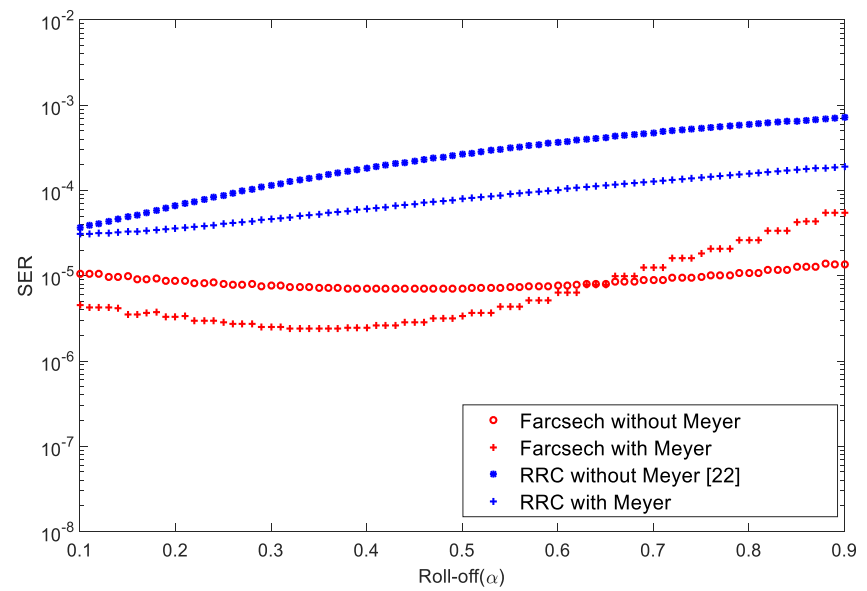

Figure 6: SER comparison of RRC and Farcsech with and without Meyer at 20 dB SNR.

Furthermore, if we apply the concept of Meyer auxiliary function on these filters given in [20]. We observe the further improvements in terms of SER as compared to without Meyer auxiliary function. The same type of comparison done in Fig.3 for $\alpha=0.1$ is considered in Fig. 4 for $\alpha=0.9$. It is clearly evident from the graphs that at $\alpha=0.9$, Farcsech pulse shaping filter is the optimal one as compared to the RRC given in [22] and Fsech pulse shaping filters but when we apply the Meyer auxiliary function on the Farcsech pulse shape filter it results in a higher SER, as compared with that without Meyer. To investigate the effect of Meyer auxiliary function on the Farcsech pulse shaping filter, in the Figs. 5 and 6 we have considered two fixed values of the Es/No 10 and $20 \mathrm{~dB}$ respectively. Then, we have plotted SER versus different values $\alpha$. It can be observed that at low values of $\alpha$ Farcsech with Meyer performs better than without Meyer while at higher values of $\alpha$ starting from $\alpha=0.65$ Farcsech without Meyer performs better than with Meyer.

Similarly in Fig. 7 it is shown SER verses $\alpha$ for the Fsech pulse shape filter with and without Meyer at 10 and $20 \mathrm{~dB}$ of Es/N0. In this case for Fsech pulse shaping filter Meyer function 


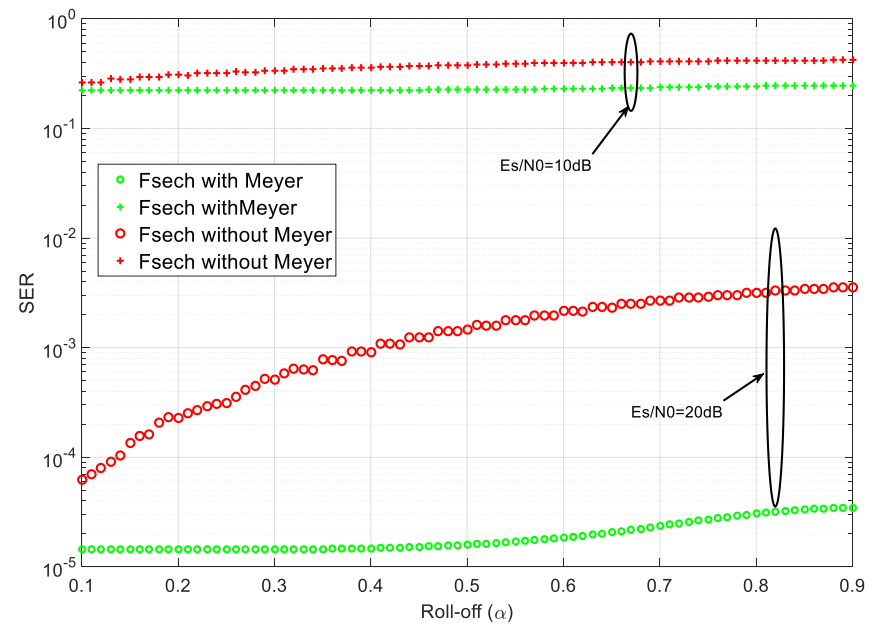

Figure 7: SER comparison of Fsech with and without Meyer at 10 and $20 \mathrm{~dB}$ SNR

always provides an improvement in comparison to Fsech pulse shaping filter.

\section{CONCLUSION}

Generalized frequency-division multiplexing is a block based multi-carrier modulation scheme that employs pulse shaping on a per subcarrier basis. The Improved Nyquist pulse shaping filters enables GFDM to achieve better performance in terms SER in comparison to raised-cosine pulse shaping filter. In this paper, we demonstrate the SER performance over the AWGN channel for GFDM using the different improved Nyquist pulse shaping filters with Meyer and Meyer auxiliary function in the case of zero forcing receiver. Form the given Figures it is clearly visible that the improved Nyquist pulse shaping filters perform better and when we include the Meyer auxiliary function in the improved Nyquist pulse shaping filters there is a further improvement in terms of SER.

\section{REFERENCES}

[1] N. Michailow, et al., "Generalized frequency division multiplexing for 5th generation cellular networks," IEEE Trans. Commun., vol. 62, no. 9, pp. 3045-306, Sept. 2014

[2] 5GNow Deliverables online resources: http://www.5gnow.eu/wpcontent/uploads/2015/04/5GNOW D3.1 v1.1 final.pdf

[3] Y. Ding, Y. Jin, L. Ren, and K. Hao, "An intelligent self-organization scheme for the internet of things," IEEE Comput. Intell. Mag., vol. 8, no. 3, pp. 41-53, Aug. 2013.

[4] G. P. Fettweis, "The Tactile Internet: Applications and Challenges," IEEE Veh. Technol. Mag., vol. 9, no. 1, pp. 64-70, Mar. 2014.

[5] N. Tadayon and S. Aissa, "Modeling and analysis of cognitive radio based IEEE 802.22 wireless regional area networks," IEEE Trans. Wireless Commun., vol. 12, no. 9, pp. 4363-4375, Sep. 2013.

[6] H. Schepker, C. Bockelmann, and A. Dekorsy, "Exploiting sparsity in channel and data estimation for sporadic multi-user communication,"
Intern. Symp. Wireless Commun. Systems, pp. 1-5, Aug. 2013, Ilmenau, Germany.

[7] Furht, Borko, and Syed A. Ahson, eds. Long Term Evolution: 3GPP LTE radio and cellular technology. Crc Press, 2016.

[8] Wunder, Gerhard, Peter Jung, Martin Kasparick, Thorsten Wild, Frank Schaich, Yejian Chen, Stephan Ten Brink et al. "5GNOW: nonorthogonal, asynchronous waveforms for future mobile applications." IEEE Communications Magazine, vol. 52, no. 2, pp. 97-105 Feb. 2014

[9] L. Barletta, M. Magarini, and A. Spalvieri, "Tight upper and lower bounds to the information rate of the phase noise channel," IEEE Intern. Symp. Inf. Theory, pp. 2284-2288, July 2013, Istanbul, Turkey.

[10] L. Barletta, M. Magarini, S. Pecorino, and A. Spalvieri, "Upper and lower bounds to the information rate transferred through first-order markov channels with free-running continuous state," IEEE Trans. Inf. Theory, vol. 60 , no. 7, pp. 3834-3844, Jul. 2014.

[11] O. H. Salim, et al., "Channel, phase noise, and frequency offset in OFDM systems: joint estimation, data detection, and hybrid Cramér-Rao lower bound," IEEE Trans. Commun., vol. 62, no. 9, pp. 3311-3325, Sept. 2014.

[12] L. Barletta, F. Bergamelli, M. Magarini, N. Carapellese, and A. Spalvieri, "Pilot-aided trellis-based demodulation," IEEE Photon. Technol. Lett., vol. 25, pp. 1234-1237, July 2013.

[13] L. Barletta, M. Magarini, and A. Spalvieri, "Staged demodulation and decoding," Optics Express, vol. 20, issue 21, pp. 23728-23734, Oct. 8, 2012.

[14] Bellanger, et al., "FBMC physical layer: a primer," PHYDYAS, January 2010

[15] L. Mendes, et al., "GFDM: providing flexibility for the 5G physical layer," Opportunities in 5G Networks: A Research and Development Perspective 2016.

[16] P. Siohan, C. Siclet, and N. Lacaille, "Analysis and design of OFDM/OQAM systems based on filterbank theory," IEEE Trans. Signal Process., vol. 50, no. 5, pp. 1170-1183, May 2002.

[17] A. Viholainen, T. Ihalainen, T. H. Stitz, M. Renfors, and M. Bellanger, "Prototype filter design for filter bank based multicarrier transmission," IEEE European Signal Process. Conference, pp. 1359-1363, 2009, Glasgow, UK.

[18] M. Matthé, N. Michailow, I. Gaspar, and G. Fettweis, "Influence of pulse shaping on bit error rate performance and out of band radiation of generalized frequency division multiplexing," IEEE Intern. Conf. Commun., pp. 43-48, 2014, Sydney, NSW.

[19] R. Datta, N. Michailow, M. Lentmaier, and G. Fettweis, "GFDM interference cancellation for flexible cognitive radio PHY design," Vehicular Technology Conference, pp. 1-5, 2012, Quebec City, QC.

[20] A. Assalini and A. M. Tonello, "Improved Nyquist pulses," IEEE Commun. Lett., vol. 8, no. 2, pp. 87-89, Feb. 2004.

[21] I. S. Gaspar, "Waveform advancements and synchronization techniques for generalized frequency division multiplexing," 2016.

[22] 5GNow Deliverables online resources: http://www.5gnow.eu/wpcontent/uploads/2015/04/5GNOW D3.2 final.pdf

[23] D. A. Guimãraes. Digital Transmission: A Simulation-Aided Introduction with VisSim/Comm. Springer Science \& Business Media, 2010.

[24] W. W. Jones and J. C. Dill, "The square root raised cosine wavelet and its relation to the Meyer functions," IEEE Trans. Signal Process., vol. 49, no. 1, pp. 248-251, Jan. 2001.

[25] I. Daubechies et al. Ten lectures on wavelets, volume 61. SIAM, 1992.

[26] Y. Meyer. Ondelettes et opérateurs: Ondelettes. Actualités mathématiques. Hermann, 1990. 